Chirurg 2013 $\cdot 84: 435$

DOI 10.1007/s00104-013-2507-y

Online publiziert: 7. April 2013

(c) Springer-Verlag Berlin Heidelberg 2013
O. Strobel · M.W. Büchler

Klinik für Allgemein-, Viszeral- und Transplantationschirurgie, Universität Heidelberg

\section{Oraler Kostaufbau ohne vorherige enterale oder parenterale Ernährung als Standard nach Pankreatoduodenektomie}

\section{Methoden}

Die Literatur wurde systematisch nach Studien durchsucht, in denen das postoperative Ernährungsregime nach $\mathrm{PD}$ untersucht wurde und die postoperative Verweildauer ein wichtiger Zielparameter war. Von 709 identifizierten Artikeln wurden 15 Studien (7 randomisiert kontrollierte Studien, 7 Kohortenstudien, eine Fall-Kontroll-Studie) mit insgesamt 3474 Patienten in die Analyse eingeschlossen. Die extrahierten Zielparameter beinhalteten Liegedauer, Dauer bis Wiedereinnahme der Normalkost, Morbidität, Inzidenz der DGE und Mortalität. Eine formelle Metaanalyse war wegen der starken Heterogenität der Studien nicht durchführbar.

\section{Ergebnisse}

Anhand der extrahierten Daten konnten 5 Ernährungswege verglichen werden: primär oraler Kostaufbau (2210 Patienten), enterale Ernährung mittels Nasojejunalsonde (165 Patienten), perkutane Gastrojejunalsonde (52 Patienten) und perkutane Jejunalsonde (623 Patien-

\begin{tabular}{|llllll}
\hline Tab. 1 & Ernährungsregime in den analysierten Studien & & \\
\hline Ernährungsregime & $\begin{array}{l}\text { Primär } \\
\text { oraler Kost- } \\
\text { aufbau }\end{array}$ & $\begin{array}{l}\text { Enteral: } \\
\text { Nasojeju- } \\
\text { nalsonde }\end{array}$ & $\begin{array}{l}\text { Enteral: per- } \\
\text { kutane Gastro- } \\
\text { jejunalsonde }\end{array}$ & $\begin{array}{l}\text { Enteral: } \\
\text { perkutane } \\
\text { Jejunalsonde }\end{array}$ & $\begin{array}{l}\text { Par- } \\
\text { en- } \\
\text { teral }\end{array}$ \\
\hline $\begin{array}{l}\text { Mittlere Verweildauer } \\
\text { (Tage) }\end{array}$ & 15 & 25 & 15 & 19 & 20 \\
\hline $\begin{array}{l}\text { Dauer bis Wiederein- } \\
\text { nahme Normalkost } \\
\text { (Tage) }\end{array}$ & 6 & 8 & 14 & 12 & 11 \\
\hline Morbidität (\%) & 49,4 & 43,8 & 75 & 56 & 50 \\
\hline Inzidenz der DGEa (\%) & 14,1 & 17,0 & 6 & 23,2 & 10,3 \\
\hline Mortalität (\%) & 4,4 & 1,8 & 2 & 4,7 & 5,4 \\
\hline DGE ,delayed gastric emptying. aUneinheitliche Definition der DGE in den verschiedenen Studien. & \\
\hline
\end{tabular}

ten) sowie totale parenterale Ernährung (TPN: 424 Patienten). Die Ergebnisse sind in - Tab. 1 zusammengefasst. Zu erwähnen ist, dass 29,4\% der Patienten mit primär oralem Kostaufbau aufgrund Komplikationen inklusive DGE vorübergehend eine TPN erhielten. - Tab. 1 zeigt, dass ein primär oraler Kostaufbau bezüglich Verweildauer und Dauer bis zur Wiedereinnahme einer normalen Kost den anderen Ernährungsregimen überlegen ist. Bezüglich Morbidität, Mortalität und Inzidenz des DGE ergaben sich keine relevanten Unterschiede; hier ist der primäre orale Kostaufbau zumindest nicht unterlegen.

\section{Fazit}

Entsprechend folgern die Autoren, dass es keine Evidenz für eine enterale oder parenterale Ernährung als Standard für den postoperativen Kostaufbau nach PD gibt. Stattdessen sollte als Standard ein primärer oraler Kostaufbau versucht werden. Nur wenn dieser wegen Komplikationen, insbesondere wegen DGE, nicht durchführbar ist, sollte auf die anderen Ernährungswege umgestiegen werden.

\section{Korrespondenzadresse}

PD Dr. O. Strobel

Klinik für Allgemein-, Viszeralund Transplantationschirurgie, Universität Heidelberg, Im Neuenheimer Feld 110, 69120 Heidelberg Oliver.Strobel@med.uni-heidelberg.de

Interessenkonflikt. Der korrespondierende Autor gibt für sich und seinen Koautor an, dass kein Interessenkonflikt besteht. 